\title{
Assessment of Human Mycotoxin Exposure in Hungary by Urinary Biomarker Determination and the Uncertainties of the Exposure Calculation: A Case Study
}

\author{
Judit Szabó-Fodor ${ }^{1, *}$, Mária Szeitzné-Szabó ${ }^{2}$, Brigitta Bóta ${ }^{1}$, Tamás Schieszl ${ }^{2}$ 'D , Cserne Angeli ${ }^{2,3}$, \\ Lucia Gambacorta ${ }^{4}$, Michele Solfrizzo ${ }^{4}$, András Szabó ${ }^{2} \mathbb{D}$ and Melinda Kovács ${ }^{1,2}$ \\ 1 Eötvös Lóránd Research Network MTA-KE-SZIE Mycotoxins in the Food Chain Research Group, \\ Kaposvár Campus, Hungarian University of Agricultural and Life Science, Guba S. u. 40, \\ 7400 Kaposvar, Hungary; bota.brigitta@uni-mate.hu (B.B.); Kovacs.Melinda@uni-mate.hu (M.K.) \\ 2 Institute of Physiology and Nutrition, Kaposvár Campus, Hungarian University of Agricultural and Life \\ Sciences, Guba S. u. 40, 7400 Kaposvar, Hungary; dr.szabomaria@gmail.com (M.S.-S.); \\ schieszltamas95@gmail.com (T.S.); Angeli.Cserne@phd.uni-mate.hu (C.A.); Szabo.Andras@uni-mate.hu (A.S.) \\ 3 Fumizol Ltd., Kisfaludy u. 6/b, 6725 Szeged, Hungary \\ 4 Institute of Sciences of Food Production (ISPA), National Research Council (CNR), 70126 Bari, Italy; \\ lucia.gambacorta@ispa.cnr.it (L.G.); michele.solfrizzo@ispa.cnr.it (M.S.) \\ * Correspondence: szabo.fodor.judit@gmail.com
}

check for updates

Citation: Szabó-Fodor, J.; Szeitzné-Szabó, M.; Bóta, B.; Schieszl,

T.; Angeli, C.; Gambacorta, L.;

Solfrizzo, M.; Szabó, A.; Kovács, M.

Assessment of Human Mycotoxin Exposure in Hungary by Urinary Biomarker Determination and the Uncertainties of the Exposure Calculation: A Case Study. Foods 2022, 11, 15. https://doi.org/ $10.3390 /$ foods 11010015

Academic Editors: Agnieszka Waśkiewicz and Marcin Bryła

Received: 9 November 2021

Accepted: 20 December 2021

Published: 22 December 2021

Publisher's Note: MDPI stays neutral with regard to jurisdictional claims in published maps and institutional affiliations.

Copyright: (C) 2021 by the authors. Licensee MDPI, Basel, Switzerland. This article is an open access article distributed under the terms and conditions of the Creative Commons Attribution (CC BY) license (https:// creativecommons.org/licenses/by/ $4.0 /)$.

\begin{abstract}
Urinary biomarkers of mycotoxin exposure were evaluated in the case of healthy people $(n=41)$ and coeliac patients $(n=19)$ by using a multi-biomarker LC-MS/MS immunoaffinity based method capable to analyse biomarkers of nine mycotoxins, i.e., fumonisin B1 (FB1), fumonisin B2 (FB2), deoxynivalenol (DON), zearalenone (ZEN), ochratoxin A (OTA), Aflatoxin B1 (AFB1), T-2 toxin, HT-2 toxin and Nivalenol (NIV). Urinary biomarker concentrations were used to calculate the probable daily intake (PDI) of fumonisin B1, deoxynivalenol, zearalenone and ochratoxin A and compared with their tolerable daily intake (TDI). The human urinary excretion rate values reported in the literature and the $24 \mathrm{~h}$ excretion rate measured in piglets were used to estimate and compare the PDI values of the four mycotoxins. The highest mean biomarker concentrations were found for DON (2.30 ng/mL for healthy people and $2.68 \mathrm{ng} / \mathrm{mL}$ for coeliac patients). Mean OTA concentration was significantly higher $(\mathrm{p}<0.001)$ in healthy people compared to coeliac patients. PDI calculated with piglets excretion data exceeded the TDI values by a much smaller percentage than when they were calculated from human data, especially for $\mathrm{FB}_{1}$. The uncertainties arising from the different calculations can be well perceived on the basis of these data.
\end{abstract}

Keywords: human exposure; mycotoxin; urine; biomarker; uncertainty; Hungary

\section{Introduction}

Mycotoxins are low molecular weight organic contaminants produced by various fungal species as secondary metabolites during growth on foods. Aflatoxins, deoxynivalenol (DON), zearalenone (ZEN), fumonisins (FBs) and ochratoxin A (OTA) are the major mycotoxins in agricultural products and food products which are monitored worldwide. These chemicals are responsible for a variety of negative health effects. It is therefore important to estimate exposure and assess the potential impact on public health.

Exposure can be determined using two different approaches, one combining indirect food consumption and contamination data and the other a direct approach based on biomarkers. In both approaches, exposure is expressed as probable daily intake (PDI). Food consumption data and prevalence data for appropriate foods are generally used to estimate population exposure. However, this method cannot estimate individual intake, it usually does not take into account all sources of contamination, so biomarker-based methods are increasingly used to assess blood or urine concentrations of dietary exposure [1,2]. The 
determination of the maximum tolerable level of mycotoxins is usually based on an estimate of the tolerable daily intake (TDI), with reference to comprehensive food consumption databases. In Europe, cereal products are the main source of exposure to mycotoxins [3], and the level of mycotoxins in the urine is an indicator of the consumption of mainly cereal products contaminated with mycotoxins. A number of studies were performed worldwide in which risk assessment was performed based on urinary biomarkers [4-9].

The multi-biomarker approach was used to validate urinary biomarkers of piglets contaminated with a mixture of DON, AFB1, FB $1, \mathrm{ZEN}$ and OTA at different concentrations. Urine samples were analysed by a multi-biomarker LC-MS/MS method developed and validated to identify and measure biomarkers of these mycotoxins. Mean percentages of dietary mycotoxins excreted in $24 \mathrm{~h}$ post-dose urine were $28.5 \%$ for DON, $36.8 \%$ for ZEN, $2.6 \% \mathrm{FB}_{1}, 2.6 \%$ for OTA and $2.5 \%$ for $\mathrm{AFB}_{1}$. A good correlation $(\mathrm{r}=0.71-0.76)$ was observed between the number of mycotoxins administered and the number of relevant biomarkers excreted in the urine $24 \mathrm{~h}$ after dosing [10]. Since then, these excretion rates have been used in many studies.

Human biomarker data are expected to provide more accurate data for estimating human exposure. A UPLC-MS/MS multi-biomarker method was used to detect and measure the presence and level of these biomarkers in urine samples from 52 volunteers living in Southern Italy [4]. For OTA and DON, 94\% and 40\% of volunteers, respectively, exceeded the tolerable daily intake of these mycotoxins. The estimated human exposure to $\mathrm{FB}_{1}$ and $\mathrm{ZEN}$ in all volunteers was well below the TDI for these mycotoxins.

In a study [5], the exposure of a German population $(n=101)$ to mycotoxins was estimated using an LC-MS/MS urinary multi-biomarker approach. Twenty-three urinary biomarkers were evaluated. Only DON and DON-GlcA (sum of DON-3-GlcA and DON-15GlcA) were detectable in quantifiable amounts due to the limited sensitivity of the analytical method. The mean daily intake of $0.52 \mu \mathrm{g}$ DON $/ \mathrm{kg}$ body weight was calculated. The results of this study suggest that the German population studied had low daily exposure to mycotoxins, but some parts of the population showed a peak above the widely accepted tolerable daily intake of DON.

The BIOMYCO study [6] was designed to assess mycotoxin exposure in Belgian volunteers using urinary biomarkers. Morning urine was collected from a representative portion of the Belgian population according to a standard study protocol in which 155 children (3-12 years of age) and 239 adults (19-65 years of age) were selected based on a random cluster sampling. These urine samples were analysed for the presence of 33 biomarkers. DON, OTA, CIT and their metabolites were the most often detected. Deoxynivalenol-15-glucuronide (DON-15-GlcA) was the main urinary DON biomarker and was found in all urine samples in a range of $\mathrm{ng} / \mathrm{mL}$. DON was detected in $70 \%$ and $37 \%$ of the samples of children and adults, respectively. Based on the urinary levels, the daily intake of DON and OTA was evaluated whereby $16-69 \%$ of the volunteers possibly exceeded the tolerable daily intake in case of DON and $1 \%$ in case of OTA.

In another work [7], a study involving a total of 300 volunteers of adults and children was conducted. OTA and DON were the most frequently occurring mycotoxins in urine, 51 and $63 \%$, respectively, in adults and 96 and 94\%, respectively, in children. The PDI values of mycotoxins were below the TDI values except for DON exposure in adults.

Franco et al. (2019) [8] aimed to assess the exposure of Brazilian residents $(n=86)$ to multiple mycotoxins and characterize the associated risk in two sampling time points. Mycotoxins in food and urine samples were determined by LCMS/MS method. Mean PDI values based on urinary biomarkers were $0.001,84.914,0.031,0.377$ and $0.002 \mu \mathrm{g} / \mathrm{kg}$ bw/day for AFB1, DON, OTA, FB1 and ZEN, respectively.

The aim of another study [9] was to investigate the exposure to mycotoxins and their association with food intake and background characteristics in adolescents of a national dietary survey. About 3000 children were included in the survey. Urine and blood samples were collected from 1105 participants for mycotoxin biomarker analysis. Mycotoxins were analysed with multi-biomarker methods in urine and serum. From the 35 analytes in 
urine, the frequency of positive samples was the following: DON 4.8\%, DON-15GlcA 9.1\%, dihydro-citrinone (DH-CIT, 0.5\%), HT-2-glucuronide (HT-2-3-GlcA, 0.1\%) and OTA 0.1\%. All probable daily intake estimates were below the TDI, except for $1.6 \%$ of the volunteers for DON.

The different studies can only be compared if the excretion rates used in the PDI calculation are taken into account. These are summarised in Table 1.

Table 1. Urinary excretion rates of mycotoxins used in human studies that assessed mycotoxin exposure from urinary mycotoxin concentrations.

\begin{tabular}{|c|c|c|c|}
\hline Human Studies & N. of Volunteers & $\begin{array}{c}\text { Excretion Rates used in the } \\
\text { Studies }\end{array}$ & References of Excretion Rate \\
\hline Solfrizzo et al., 2014 [4] & 52 & $\begin{array}{l}\text { total OTA } 2.6 \% \\
\mathrm{FB}_{1} 2.6 \% \\
\text { total ZEN } 36.8 \% \\
\mathrm{AFB}_{1} \text { as } \mathrm{AFM}_{1} 2.5 \% \\
\text { total DON } 27.9 \% \\
\text { total DON } 50 \% \\
\mathrm{FB}_{1} 0.5 \%\end{array}$ & Gambacorta et al., 2013 [10] (piglets) \\
\hline Gerding et al., 2014 [5] & 101 & total DON 68\% & Warth et al., 2013 [12] (humans) \\
\hline $\begin{array}{l}\text { Heyndrickx et al., } 2015 \text { [6] } \\
\text { Vidal et al., } 2018 \text { [14] }\end{array}$ & $\begin{array}{c}394 \\
30\end{array}$ & $\begin{array}{c}\text { total DON 72\% } \\
\text { DON+DON-3-GLC 72\% } \\
\text { OTA } 2.6 \%\end{array}$ & $\begin{array}{c}\text { Turner et al., } 2010 \text { [13] (humans) } \\
\text { Turner et al., } 2010 \text { [13] (humans) } \\
\text { Gambacorta et al., } 2013 \text { [10] (piglets) }\end{array}$ \\
\hline Mitropoulou et al., 2018 [7] & 300 & $\begin{array}{l}\text { total DON } 72 \% \\
\mathrm{FB}_{1} 0.5 \% \\
\text { ZEN } 28.4 \% \\
\text { total ZEN } 36.8 \%\end{array}$ & $\begin{array}{l}\text { Turner et al., } 2010 \text { [13] (humans) } \\
\text { Riley et al., } 2012 \text { [15] (humans) } \\
\text { Gambacorta et al., } 2013 \text { [10] (piglets) }\end{array}$ \\
\hline Fan et al., 2019 [16] & 260 & $\begin{array}{c}\text { total DON } 72 \% \\
\text { AFB }_{1} \text { as } \mathrm{AFM}_{1} 1.5 \% \\
\text { OTA } 2.5 \% \\
\text { FB }_{1} 1 \% \\
\text { ZEN } 9.4 \%\end{array}$ & $\begin{array}{l}\text { Turner et al., } 2010 \text { [13] (humans) } \\
\text { Zhu et al., } 1987 \text { [17] (human) } \\
\text { Degen } 2016 \text { [18] (human) } \\
\text { Riley et al., } 2012 \text { [15] (humans) } \\
\text { Warth et al., } 2013 \text { [12] (humans) }\end{array}$ \\
\hline Franco et al., 2019 [8] & 86 & $\begin{array}{c}\mathrm{AFB}_{1} \text { as } \mathrm{AFM}_{1} \text { for women } 1.5 \% \\
\mathrm{AFB}_{1} \text { as } \mathrm{AFM}_{1} \text { for men } 1.7 \% \\
\text { total DON for women } 72 \% \\
\text { total DON for men } 50 \% \\
\mathrm{FB}_{1} 0.5 \% \\
\mathrm{ZEN} 36.8 \%\end{array}$ & $\begin{array}{l}\text { Zhu et al., } 1987 \text { [17] (humans) } \\
\text { Zhu et al., } 1987 \text { [17] (humans) } \\
\text { Vidal et al., } 2018 \text { [14] (humans) } \\
\text { Vidal et al., } 2018 \text { [14] (humans) } \\
\text { Riley et al., } 2012 \text { [15] (humans) } \\
\text { Gambacorta et al., } 2013 \text { [10] (piglets) }\end{array}$ \\
\hline Lemming et al., 2019 [9] & 1105 & DON+DON-15-GLC 72\% & Turner et al., 2010 [13] (humans) \\
\hline
\end{tabular}

Maize-derived ingredients are frequently used in food formulation. In addition, some population sub-groups (vegans and coeliac persons) may be more exposed compared to the general population.

In this paper, we report the results on the occurrence of DON, OTA, ZEN and $\mathrm{FB}_{1}$ in urine samples of 60 (41 healthy and 19 coeliac patients) volunteers. A probable daily intake was calculated for these mycotoxins and compared to the established tolerable daily intake to uncover potential risks among Hungarian adults and children.

\section{Materials and Methods}

\subsection{Institutional Review Board Statement}

The study was conducted in accordance with the Declaration of Helsinki, and the protocol was approved by the Scientific and Research Ethical Committee of the Medical Research Council (Hungary) (ID 32958-3/2017/EKU) and included informed, written consent of all patients. 


\subsection{Participants and Urine Collection}

For the evaluation of human exposure to DON, FB1, ZEN and OTA, 60 individuals (41 healthy and 19 coeliac patients) residing in Somogy, Baranya and Pest counties (Hungary) were invited to participate in the urine sampling. Before starting the experiment, they were invited to answer general questions about their health status. People with signs and/or symptoms of liver or kidney illness or any chronic disease were not included in the study due to potential interferences with the metabolism of mycotoxins and creatinine.

Each individual was asked to provide a $24 \mathrm{~h}$ urine sample.

All samples were subsequently stored at $-20{ }^{\circ} \mathrm{C}$ until transportation for biomarker analysis.

Frozen samples were sent, under dry ice, to the Institute of Sciences of Food Production (ISPA) in Bari (Italy) for mycotoxins (ZEN, DON, FB1, fumonisin B2, OTA, T-2 toxin, HT-2 toxin and nivalenol) and metabolites (deepoxy-deoxynivalenol, $\alpha$-zearalenol, $\beta$-zearalenol, aflatoxin M1) determination.

\subsection{Analysis of Urinary Biomarkers}

The following mycotoxins and metabolites were analysed in urine samples: ZEN, DON, deepoxy-deoxynivalenol (DOM-1), FB1, fumonisin B2 (FB2), OTA, $\alpha$-zearalenol $(\alpha-Z O L), \beta$-zearalenol ( $\beta$-ZOL), aflatoxin M1 (AFM1), T-2 toxin, HT-2 toxin and nivalenol (NIV).

Urine samples were thawed, centrifuged and analysed by using the ultra-performance liquid chromatography-tandem mass spectrometry (LC-MS/MS) method previously described $[4,19]$. Briefly, $6 \mathrm{~mL}$ urine was enzymatically digested with $\beta$-glucuronidase/sulfatase type H-2 from Helix pomatia (Sigma Aldrich, Milan, Italy) to hydrolyse glucuronide and sulphate conjugates of mycotoxins and/or their metabolites into free mycotoxins/metabolites. Then the digested urine was diluted with water and purified on a Myco6in $1+{ }^{\mathrm{TM}}$ multiantibody immunoaffinity column (IAC, Vicam, Watertown, MA, USA) connected in tandem with an OASIS ${ }^{\circledR}$ HLB column (Waters, Milford, MA, USA). Therefore, the sample passed through the immunoaffinity column and then through the OASIS column. After complete elution of the sample, the two columns were separated, washed and the analytes were eluted separately from each column. The two purified extracts were gathered together, dried, reconstituted with $200 \mu \mathrm{L}$ of a mixture of methanol:water:acetic acid (20:80:0.5) and analysed by LC-MS/MS. Matrix matched calibration curves were used for LC-MS/MS analyte quantitation in the purified urine extracts. The analysis was performed on a triple quadrupole API 5000 mass spectrometer (Applied Biosystems, Foster City, CA, USA), equipped with an ESI interfaced with an Acquity UPLC system comprising a binary pump and a microautosampler (Waters). Data acquisition and processing were performed with Analyst version 1.5.1 software (Applied Biosystems). More details, together with chromatographic and mass spectrometric operating conditions, are described elsewhere $[4,20]$.

\subsection{Creatinine Analysis in Human Urine}

Creatinin was determined with a Roche/Hitachi cobas c 501/502 instrument using the Creatinine Jaffé Gen.2 700 test (Roche Diagnostics GmbH, Mannheim, Germany) in the urine samples.

This kinetic colorimetric assay is based on the Jaffe method. In alkaline solution, creatinine is a yellow-orange complex with picric acid forms. The degree of dye formation is proportional to the creatine concentration of the sample. The test procedure uses a 'blind measurement' of it in order to minimise the interference caused by bilirubin reduction.

\subsection{Statistical Analysis}

Statistical analyses were performed using Microsoft Office Excel (2013) and IBM SPSS 20.0 (2012) software. Data processing and the mathematical-statistical calculations were performed using the compare means (Independent Samples $t$-Test, one-way ANOVA with Tukey's post-hoc test), correlations and descriptive statistics modules. 
A value of the limit of detection (LOD)/2 was used for not detected analytes, whereas for values $>$ LOD and <limit of quantification (LOQ), a value of LOQ/2 was used.

The mycotoxin concentrations were normalised with the creatinine level (mycotoxin concentration/creatinine concentration).

\subsection{Exposure Assessment}

The urinary biomarker concentrations measured in this study were used to estimate the probable daily intake (PDI) of each mycotoxin according to the following formula [4].

$$
\mathrm{PDI}=\mathrm{C} \times \frac{\mathrm{V}}{\mathrm{W}} \times \frac{100}{\mathrm{E}}
$$

PDI probable daily intake of mycotoxin ( $\mu \mathrm{g} / \mathrm{kg}$ body weight);

where:

C human urinary biomarker concentration $(\mu \mathrm{g} / \mathrm{L})$;

V 24 h human urine volume measured for each volunteer (L);

W human body weight measured for each volunteer $(\mathrm{kg})$;

E mean urinary excretion rate of mycotoxin *.

* The calculations were performed in two ways, taking 'pigs' and 'humans' excretions rates into consideration:

- $\quad$ Data derived after $24 \mathrm{~h}$ post dose in piglets ([10]: 36.8\% for ZEN, $27.9 \%$ for DON, $2.6 \%$ for FB1, 2.6\% for OTA);

- $\quad$ Excretion rates from human studies: $72.3 \%$ for DON [13]; 0,5\% for FB1 [15]; 9,4\% for ZEN [12]; 2.5\% for OTA [18,21].

\section{Results}

In total, 60 urine samples from healthy people and coeliac patients living in Hungary were analysed for the presence of 12 urinary mycotoxins and/or their metabolites. Six out of twelve analytes were detected whereby ZEN, DON, FB1, FB2 and OTA were the most frequently detected. The following mycotoxins and metabolites could not be detected or quantified: DOM-1, AFM1, T-2 toxin and HT-2 toxin. NIV was detected in only one sample.

The concentrations of these mycotoxins are presented in Table 2. The creatininenormalised toxin concentrations are shown in Table 3.

The maximum biomarker concentrations were found in healthy people, i.e., $26.731 \mathrm{ng} / \mathrm{mg}$ of DON followed by FB1 (1.764 ng/mg), OTA (0.582 ng/mg) and ZEN $(0.504 \mathrm{ng} / \mathrm{mg})$. From Table 4 , it is also evident that urinary concentrations of DON are much higher than those of the other biomarkers.

A significant difference between the two groups of volunteers was experienced only for OTA concentrations ( $\mathrm{p}<0.001)$.

In our data, the DON concentration means the sum of free DON plus DON derived from DON-3-glucuronide (DON-3-GlcA) and DON-15-glucuronide (DON-15-GlcA), total $\mathrm{ZEN}$ means the sum of urinary concentrations of free $\mathrm{ZEN}+\alpha-\mathrm{ZOL}+\beta-\mathrm{ZOL}$ plus their glucuronide and sulphate conjugates and OTA means the sum of free OTA plus its glucuronide conjugate.

The estimated daily mean intake (probable daily intake) of the four investigated mycotoxins were calculated and are reported in Tables 4 and 5. Due to the availability of human excretion rate for the four mycotoxins considered in this study we used the human excretion rate values found in the literature (i.e., $72.3 \%$ for DON [13], 0.5\% for FB1 [15], 9.4\% for ZEN [12] and 2.5\% for OTA [18,21]. We compared the PDI data obtained from the human excretion rate to those obtained using the $24 \mathrm{~h}$ excretion rate measured in piglets [10]. 
Table 2. Concentration of mycotoxins $(\mathrm{ng} / \mathrm{mL})$ in the urine samples of healthy people and coeliac patients.

\begin{tabular}{|c|c|c|c|c|c|c|}
\hline & $\begin{array}{c}n \\
\text { Positive }\end{array}$ & Mean & Median & SD * & Minimum & Maximum \\
\hline \multicolumn{7}{|c|}{ Healthy people } \\
\hline ZEN & 40 & 0.042 & 0.029 & 0.037 & 0.012 & 0.174 \\
\hline$\alpha-\mathrm{ZOL}$ & 34 & 0.014 & 0.013 & 0.012 & 0.004 & 0.063 \\
\hline$\beta-\mathrm{ZOL}$ & 8 & 0.006 & 0.004 & 0.006 & 0.013 & 0.033 \\
\hline Total ZEN & 41 & 0.062 & 0.048 & 0.048 & 0.008 & 0.199 \\
\hline $\mathrm{DON}$ & 18 & 2.302 & 0.063 & 3.734 & 2.010 & 18.947 \\
\hline $\mathrm{FB}_{1}$ & 41 & 0.267 & 0.194 & 0.246 & 0.141 & 1.525 \\
\hline $\mathrm{FB}_{2}$ & 41 & 0.060 & 0.032 & 0.134 & 0.008 & 0.875 \\
\hline OTA & 41 & $0.166^{\mathrm{a}}$ & 0.124 & 0.155 & 0.071 & 1.024 \\
\hline NIV & 1 & 0.475 & 0.475 & & 0.475 & 0.475 \\
\hline \multicolumn{7}{|c|}{ Coeliac patients } \\
\hline ZEN & 19 & 0.041 & 0.034 & 0.030 & 0.011 & 0.121 \\
\hline$\alpha-\mathrm{ZOL}$ & 11 & 0.013 & 0.010 & 0.018 & 0.004 & 0.071 \\
\hline$\beta-\mathrm{ZOL}$ & 2 & 0.005 & 0.004 & 0.003 & 0.013 & 0.013 \\
\hline Total ZEN & 19 & 0.059 & 0.040 & 0.044 & 0.022 & 0.152 \\
\hline $\mathrm{DON}$ & 9 & 2.681 & 0.063 & 3.095 & 3.876 & 9.484 \\
\hline $\mathrm{FB}_{1}$ & 19 & 0.229 & 0.219 & 0.055 & 0.146 & 0.356 \\
\hline $\mathrm{FB}_{2}$ & 19 & 0.046 & 0.029 & 0.046 & 0.016 & 0.226 \\
\hline OTA & 19 & $0.089^{b}$ & 0.078 & 0.044 & 0.052 & 0.243 \\
\hline
\end{tabular}

$\overline{a, b}$ Numbers with different superscripts indicate significant differences $(\mathrm{p} \leq 0.001)$ between groups.* SD standard deviation. DOM-1, AFM1, T-2 toxin and HT-2 toxin were negative in all the samples (LOD DOM-1: $0.136 \mathrm{ng} / \mathrm{mL}$; LOD AFM1: $0.019 \mathrm{ng} / \mathrm{mL}$; LOD T-2 toxin: $0.250 \mathrm{ng} / \mathrm{mL}$; LOD HT-2 toxin: $6.250 \mathrm{ng} / \mathrm{mL}$; LOQ DOM-1: $0.45 \mathrm{ng} / \mathrm{mL}$; LOQ AFM1: $0.063 \mathrm{ng} / \mathrm{mL}$; LOQ T-2 toxin: $0.830 \mathrm{ng} / \mathrm{mL}$; LOQ HT-2 toxin: $20.8 \mathrm{ng} / \mathrm{mL}$ ) LOD ZEN: $0.001 \mathrm{ng} / \mathrm{mL}$ LOQ ZEN: $0.003 \mathrm{ng} / \mathrm{mL}$; LOD $\alpha$-ZOL: $0.002 \mathrm{ng} / \mathrm{mL}$, LOQ $\alpha$-ZOL: $0.007 \mathrm{ng} / \mathrm{mL}$; LOD $\beta$-ZOL: $0.008 \mathrm{ng} / \mathrm{mL}$, LOQ $\beta$-ZOL: $0.025 \mathrm{ng} / \mathrm{mL}$, LOD DON: $0.13 \mathrm{ng} / \mathrm{mL}$, LOQ DON: $0.42 \mathrm{ng} / \mathrm{mL} ;$ LOD FB1: $0.005 \mathrm{ng} / \mathrm{mL}$, LOQ FB1: $0.017 \mathrm{ng} / \mathrm{mL}$, LOD FB2: $0.005 \mathrm{ng} / \mathrm{mL}$, LOQ FB2: $0.016 \mathrm{ng} / \mathrm{mL}$; LOD OTA: $0.0003 \mathrm{ng} / \mathrm{mL}$, LOQ OTA: $0.001 \mathrm{ng} / \mathrm{mL}$ LOD NIV: $0.018 \mathrm{ng} / \mathrm{mL}$, LOQ NIV: $0.06 \mathrm{ng} / \mathrm{mL}$

Table 3. Creatinine normalised toxin concentrations $(\mathrm{ng} / \mathrm{mg})$ in urine samples of healthy people and coeliac patients.

\begin{tabular}{|c|c|c|c|c|c|c|c|}
\hline & & $\begin{array}{c}n \\
\text { Positive }\end{array}$ & Mean & Median & SD * & Minimum & Maximum \\
\hline \multirow{4}{*}{$\begin{array}{c}\text { Healthy } \\
\text { people }\end{array}$} & Total ZEN & 41 & 0.105 & 0.063 & 0.115 & 0.0104 & 0.504 \\
\hline & DON & 18 & 3.632 & 0.181 & 6.495 & 0.034 & 26.731 \\
\hline & $\mathrm{FB}_{1}$ & 41 & 0.413 & 0.278 & 0.246 & 0.069 & 1.764 \\
\hline & OTA & 41 & 0.218 & 0.192 & 0.104 & 0.083 & 0.582 \\
\hline \multirow{4}{*}{$\begin{array}{l}\text { Coeliac } \\
\text { patients }\end{array}$} & ZEN & 19 & 0.101 & 0.064 & 0.110 & 0.017 & 0.371 \\
\hline & DON & 9 & 3.540 & 0.147 & 5.653 & 0.043 & 23.752 \\
\hline & $\mathrm{FB}_{1}$ & 19 & 0.341 & 0.299 & 0.173 & 0.129 & 0.793 \\
\hline & OTA & 19 & 0.128 & 0.100 & 0.070 & 0.039 & 0.332 \\
\hline
\end{tabular}

* SD standard deviation. 
Table 4. Mean and maximum concentration of mycotoxins in human urine samples collected in Hungary from healthy people and coeliac patients and estimated PDI values based on the urinary excretion rate for piglets [10].

\begin{tabular}{|c|c|c|c|c|c|c|c|c|c|c|c|c|}
\hline & & \multirow{2}{*}{ Positive } & \multicolumn{2}{|c|}{ Conc. $(\mu \mathrm{g} / \mathrm{L})$} & \multirow{2}{*}{$\begin{array}{l}\text { Pig Urinary } \\
\text { Excretion }(\%)\end{array}$} & \multicolumn{2}{|c|}{ PDI ( $\mu g / k g$ bw/day) } & \multirow{2}{*}{$\begin{array}{c}\text { TDI* ( } \mu \mathrm{g} / \mathrm{kg} \\
\text { bw/day) }\end{array}$} & \multicolumn{2}{|c|}{ PDI/TDI \% } & \multirow{2}{*}{$\begin{array}{l}\text { n. above } \\
\text { TDI }\end{array}$} & \multirow{2}{*}{$\begin{array}{c}\% \text { above } \\
\text { TDI }\end{array}$} \\
\hline & & & Mean & Maximum & & Mean & Maximum & & Mean & Maximum & & \\
\hline \multirow{4}{*}{$\begin{array}{c}\text { Healthy } \\
\text { people } \\
n=41\end{array}$} & Total ZEN & 41 & 0.062 & 0.199 & 36.8 & 0.004 & 0.020 & 0.25 & 1.6 & 8.0 & 0 & 0 \\
\hline & $\mathrm{DON}$ & 18 & 2.302 & 18.947 & 27.9 & 0.195 & 1.617 & 1.00 & 19.5 & 161.7 & 2 & 4.8 \\
\hline & $\mathrm{FB}_{1}$ & 41 & 0.267 & 1.525 & 2.6 & 0.225 & 1.062 & 1.00 & 22.5 & 106.2 & 1 & 2.4 \\
\hline & OTA & 41 & 0.166 & 1.024 & 2.6 & 0.144 & 1.055 & 0.017 & 847.1 & 6205.9 & 41 & 100 \\
\hline \multirow{4}{*}{$\begin{array}{c}\text { Coeliac } \\
\text { patients } \\
n=19\end{array}$} & Total ZEN & 19 & 0.059 & 0.152 & 36.8 & 0.004 & 0.015 & 0.25 & 1.6 & 6.0 & 0 & 0 \\
\hline & DON & 9 & 2.681 & 9.484 & 27.9 & 0.224 & 1.307 & 1.00 & 22.4 & 130.7 & 1 & 5.3 \\
\hline & $\mathrm{FB}_{1}$ & 19 & 0.229 & 0.356 & 2.6 & 0.215 & 0.410 & 1.00 & 21.5 & 41.0 & 0 & 0 \\
\hline & OTA & 19 & 0.089 & 0.243 & 2.6 & 0.089 & 0.374 & 0.017 & 523.5 & 2200.0 & 19 & 100 \\
\hline
\end{tabular}

${ }^{*}$ TDI values for mycotoxins: ZEN $0.250 \mu \mathrm{g} / \mathrm{kg}$ bw/day [22]; FB1 $1.0 \mu \mathrm{g} / \mathrm{kg}$ bw/day [23]; DON $1.0 \mu \mathrm{g} / \mathrm{kg}$ bw/day [13]; OTA 0.017 $\mu \mathrm{g} / / \mathrm{kg}$ bw/day (this value derived from 0.120 $\mu \mathrm{g} / \mathrm{kg}$ bw/week) [24].

Table 5. Mean and maximum concentration of mycotoxins in human urine samples collected in Hungary from healthy people and coeliac patients and estimated PDI values based on the urinary excretion rate of human studies.

\begin{tabular}{|c|c|c|c|c|c|c|c|c|c|c|c|c|}
\hline & & \multirow{2}{*}{ Positive } & \multicolumn{2}{|c|}{ Conc. $(\mu g / L)$} & \multirow{2}{*}{$\begin{array}{l}\text { Human Uinary } \\
\text { Excretion (\%) }\end{array}$} & \multicolumn{2}{|c|}{ PDI ( $\mu g / k g$ bw/day) } & \multirow{2}{*}{$\begin{array}{c}\text { TDI * }(\mu g / g \\
\text { bw/day) }\end{array}$} & \multicolumn{2}{|c|}{ PDI/TDI \% } & \multirow{2}{*}{$\begin{array}{c}\text { n above } \\
\text { TDI }\end{array}$} & \multirow{2}{*}{$\begin{array}{c}\% \text { above } \\
\text { TDI }\end{array}$} \\
\hline & & & Mean & Maximum & & Mean & Maximum & & Mean & Maximum & & \\
\hline \multirow{4}{*}{$\begin{array}{c}\text { Healthy } \\
\text { people } \\
n=41\end{array}$} & Total ZEN & 41 & 0.062 & 0.199 & 9.4 & 0.016 & 0.080 & 0.25 & 6.4 & 32.0 & 0 & 0 \\
\hline & $\mathrm{DON}$ & 18 & 2.302 & 18.947 & 72.3 & 0.075 & 0.624 & 1.00 & 7.5 & 62.4 & 0 & 0 \\
\hline & $\mathrm{FB}_{1}$ & 41 & 0.267 & 1.525 & 0.5 & 1.172 & 5.524 & 1.00 & 117.2 & 552.4 & 16 & 39.0 \\
\hline & OTA & 41 & 0.166 & 1.024 & 2.5 & 0.150 & 1.097 & 0.017 & 882.3 & 6452.9 & 41 & 100 \\
\hline \multirow{4}{*}{$\begin{array}{c}\text { Coeliac } \\
\text { patients } \\
n=19\end{array}$} & Total ZEN & 19 & 0.059 & 0.152 & 9.4 & 0.016 & 0.061 & 0.25 & 6.4 & 24.4 & 0 & 0 \\
\hline & DON & 9 & 2.681 & 90484 & 72.3 & 0.087 & 0.505 & 1.00 & 8.7 & 50.5 & 0 & 0 \\
\hline & $\mathrm{FB}_{1}$ & 19 & 0.229 & 0.356 & 0.5 & 1.116 & 2.133 & 1.00 & 111.6 & 213.3 & 12 & 63.2 \\
\hline & OTA & 19 & 0.089 & 0.243 & 2.5 & 0.092 & 0.389 & 0.017 & 541.2 & 2288.2 & 19 & 100 \\
\hline
\end{tabular}

* TDI values: see the note of Table 4 . 
Based on piglets excretion of FB1 [10], 2.4\% of PDI values in healthy people were above its TDI. No coeliac patients were above the TDI for FB1 (Table 4). Much higher percentages of PDI above the TDI of FB1 were found when the human excretion rate of FB1 was used for calculations. In particular, 39.0\% of the PDI values in healthy people and $63.0 \%$ in coeliac patients were above the TDI of FB1 (Table 5).

According to the excretion rate of piglets, the mean PDI of FB1 was $22.5 \%$ of its TDI in healthy people and $21.5 \%$ in coeliac patients (Table 4 ). In contrast, based on the human excretion rate, the mean PDI of FB1 was $117.2 \%$ of its TDI in healthy people and $111.6 \%$ in coeliac patients (Table 5).

The PDI values of ZEN were above its TDI is $0 \%$ of healthy people and for coeliac patients according to piglets and humans excretion rates (Tables 4 and 5). The mean PDI of DON was $19.5 \%$ of its TDI in healthy people and $22.4 \%$ in coeliac patients taking into account the piglets excretion rate (Table 4).

When using the human excretion rate, the mean PDI of DON was only $7.5 \%$ of its TDI in healthy people (8.7\% for coeliac people) (Table 5$)$.

In $1.7 \%$ of all subjects studied $(n=60)$, the PDI of FB1 was greater than its TDI by using the excretion rate of piglets and $46.7 \%$ by using the human excretion rate, which is a difference of twenty-seven times (Tables 4 and 5).

As reported in Tables 4 and 5, similar mean values of PDI of OTA were estimated in healthy people by using piglets and human excretion rates, i.e., 0.144 and $0.150 \mu \mathrm{g} / \mathrm{kg}$ body weight, respectively. The values of PDI of OTA in coeliac patients were also similar by using piglets and human excretion rates, i.e., 0.089 and $0.092 \mu \mathrm{g} / \mathrm{kg}$ body weight, respectively. These values were about 5-9 times higher than the TDI $(0.017 \mu \mathrm{g} / \mathrm{kg}$ body weight) established for this mycotoxin, and $100 \%$ of volunteers participating in our study exceeded the TDI of OTA regardless of the excretion rate used for PDI calculation. It should be noted that the piglet and human excretion rates used in this study are very similar, i.e., $2.6 \%$ and $2.5 \%$, respectively $[10,18]$. Moreover, the mean PDI values of coeliac patients were lower than those calculated in healthy people, which was statistically significant for OTA $(\mathrm{p}<0.0001)$.

\section{Discussion}

The calculated values of PDI strongly depended on the urinary excretion rate selected for each mycotoxin with the exception of OTA since similar rates were reported for piglets $(2.6 \%)$ and humans $(2.5 \%)[10,18]$. A large number of values above the TDI of FB1 were observed especially when the human excretion rate was used for PDI calculation. The human mean urinary excretion rate of FB1, reported by Riley et al. (2012) [15], is 0.5\% of ingested FB1 measured in eight volunteers. Thus, total urinary excretion was less than $1 \%$ of the cumulative dose $(0.12-0.9 \%)$, which was still higher than that reported by van der Westhuizen et al. (2011) [25], i.e., 0.075\% (0.054-0.104\%). These values are much less than $2.6 \%$ reported in $24 \mathrm{~h}$ urine of four piglets fed one bolus of contaminated feed [10].

Therefore, the percentage of total volunteers $(n=60)$ with PDI $>$ TDI of FB1 was $1.7 \%$ or $46.7 \%$ when the considered excretion rate was $2.6 \%$ or $0.5 \%$, respectively. In the calculations, we took into account the stricter $1.0 \mu \mathrm{g} / \mathrm{kg}$ bw $/$ day [23] recommendation for the value of TDI. These values would be significantly lower if the WHO/JECFA recommendation (2017) $(2 \mu \mathrm{g} / \mathrm{kg}$ bw/day) were taken into account.

As an example, D'Arco et al. (2009) [26] considered the FBs occurrence in products addressed to children or vegans and coeliac persons on the Italian and Spanish markets, reporting a higher incidence of positive samples within the class of organic foods [26]. Among population categories particularly vulnerable to mycotoxins, patients suffering from coeliac disease might be potentially overexposed due to their restricted gluten-free (GF) diet.

Based on human data, the excretion rate of total DON and total ZEN is 72.3\% [13] and $9.4 \%$ [12], respectively, while these values are $27.9 \%$ for total DON and $36.8 \%$ for total ZEN according to a piglet study [10]. 
Gerding et al. (2014) [5] measured mycotoxin exposure in the German population by using a direct method, i.e., measurement of free and conjugated mycotoxins and their metabolites since they did not hydrolyse the urine samples. The most common biomarker of DON was DON-GlcA that was present in $82 \%$ of samples, followed by free DON that was present in $29 \%$ of the samples. An excretion rate of $68 \%$ of total DON was used to calculate the PDI, according to Warth et al. (2013) [12]. This workgroup [12] conducted their study on the kinetics of DON and ZEN in a single healthy 27-year-old male volunteer. The volunteer consumed high-grain meals for 4 days, and urine was collected at a 24-h period. The working group determined a toxin excretion rate of $68 \%(60-71 \%)$ for total DON (15\% free DON, 14\% DON-3-GlcA and 39\% DON-15-GlcA), and this value for ZEN was $9.4 \%(7-13.2 \%)$. Thus, the excretion rate of free DON was determined to be $15 \%$, while the clearance rate of free ZEN was estimated to be $9.4 \%$, but only from data of a single volunteer $(\mathrm{n}=1)$.

In a human study [13] of 35-, 21- and 59-year-old volunteers monitored the first morning urine for 12 days and urine samples were hydrolysed before analysis. The total $\mathrm{DON}$ (free DON + glucuronised DON) excretion rate was $72.3 \%(59.1-85.5 \%$ ). This is similar to $68 \%$ measured by Warth et al. (2013) [12] for total DON but different from the $27.9 \%$ reported for piglets [10].

Franco et al. (2019) [8] estimated human exposure in the Brazilian population. The total DON excretion rates considered for women and men were $72 \%$ and $50 \%$, respectively (based on [14]); 50\% for OTA (based on [27]), $0.5 \%$ for FB1 (based on [15]) and 36.8\% for ZEN (based on [10]). Thus, results from human and animal experiments were used in the PDI calculation.

Vidal et al. (2018) [14] found a discrepancy between the total DON excretion rate of women and men and free DON was set at $25 \%$, which is much higher than the $15 \%$ reported by Warth et al. (2013) [12].

Studer-Rohr et al. (2000) [21] determined the kinetics of OTA involving a single healthy volunteer. About 3\% of a single oral dose of tritium-labelled OTA was excreted with the daily urine by a male human during the first 6 days, with a total urinary excretion of $62 \%$ of the dose after 75 days.

In the case of free OTA, there is no clearly defined clearance rate in humans. Along with missing data on the temporal variability and possible dose-related effects of renal excretion of OTA in humans, the uncertainty in calculating dietary intake based on biomarker levels is very high for this mycotoxin [18].

Attempts were made to estimate daily intake of OTA based on urinary concentrations in spot samples, but the results are considered questionable because of long half-life, strong plasma protein binding and uncertain excretion rates (values from $2.6 \%$ to $50 \%$ were used) $[4,6-8,18]$.

According to Solfrizzo et al. (2014) [4], in Italy, the mean value of estimated PDI $(0.139 \mu \mathrm{g} / \mathrm{kg}$ body weight) based on excretion value of piglets is 5.8-127 times higher than the European PDI values estimated with the diet approach. Our data and the calculated values are in great agreement with the data of Solfrizzo et al. (2014) [4]. These data clearly show that the estimated human exposure to OTA is higher when using the biomarker approach compared to the diet approach. According to Solfrizzo et al. (2014) [4], there are two possible explanations for this: (a) the human excretion of OTA is completely different from that of piglets; (b) food diet approach do not cover all sources of OTA exposure.

The largest differences are observed for FB1 and that for ZEN, all the PDI values did not exceed the TDI value of this mycotoxin by using both human and porcine excretion rates. Furthermore, in the case of OTA, all PDI values exceeded the TDI of this mycotoxin in all cases by using both human and porcine excretion rates. The $\%$ of samples with PDI > TDI is $100 \%$ for OTA and $0 \%$ for ZEN.

As an international comparison, our data can be compared with the following studies:

In the study of Solfrizzo et al., 2014 [4], 94\% and 40\% of the Italian volunteers exceeded the TDI of OTA and DON, respectively, whereas human exposure to FB1 and ZEN was 
largely below the TDI of these mycotoxins for all volunteers. In our case, 100\% for OTA, $5.0 \%$ for DON and $1.7 \%$ for FB1 of volunteers exceeded the TDI values using porcine excretion rate. However, it should be noted that Solfrizzo et al. (2014) [4] considered the milder recommendation ( $2 \mu \mathrm{g} / \mathrm{kg}$ bw $/$ day) regarding the TDI value of FB1, whereas we used a TDI of $1 \mu \mathrm{g} / \mathrm{kg}$ bw/day.

In the study of Gerding et al. (2014) [5], a PDI of $0.52 \mu \mathrm{g}$ DON $/ \mathrm{kg}$ body weight was calculated by using a human clearance rate of $68 \%$ for DON. In our case, taking into account a human excretion rate of $72.3 \%$, the calculated PDI of DON was lower, i.e., $0.075 \mu \mathrm{g} / \mathrm{kg}$ $\mathrm{bw} /$ day for healthy people and $0.087 \mu \mathrm{g} / \mathrm{kg} \mathrm{bw} /$ day for coeliac people.

According to Lemming et al. (2019) [9], in Sweden, based on 1105 urine samples, all PDI estimates were below tolerable daily intakes, except for $1.6 \%$ of the participants for DON. According to our data, in the case of Hungarian participants, the PDI of $5 \%$ of volunteers exceeds the TDI of DON.

Based on the above, it can be observed that it is worth comparing the various excretion rates published in the literature so far, as even a low percentage difference can result in multiple exposure levels, e.g., for FB1 (0.5\% based on [15] or 2.6\% based on [10]).

\section{Conclusions}

A multi-mycotoxin exposure was found for all tested volunteers participating in the study. This is the first report on the occurrence of urinary FB1, ZEN, DON and OTA in Hungary.

The estimated PDI values of FB1 largely exceeded the TDI value for this mycotoxin. PDI values are above TDI in 39.0\% (63.2\% for coeliac patients) in the case of FB1, using the human excretion rate. We found drastic differences in the excretion rates used in the literature and used them to point out how much difference it can cause in the PDI calculation.

With this comparison, we would like to shed light on the direction and need for further research on validated excretion rates and the uncertainties of the exposure calculated in this way.

For plant farmers and the food industry, our practical advice is to use the fungicide concentration and treatment frequency according to the providers' guidance in practice to prevent mycotoxin-producing mould contamination. It is recommended to keep dry, cool conditions during the storage of cereals.

Author Contributions: Conceptualization, M.K.; methodology, M.S. and L.G.; validation, M.S. and L.G.; formal analysis, B.B., M.S.-S., T.S. and C.A.; investigation, B.B., M.S.-S., T.S. and C.A.; resources, M.K.; data curation, B.B., M.S.-S., T.S. and C.A.; writing - original draft preparation, J.S.-F., M.S. and M.S.-S.; writing - review and editing, J.S.-F. and A.S.; supervision, A.S.; project administration, A.S. and M.K.; funding acquisition, M.K. All authors have read and agreed to the published version of the manuscript.

Funding: This research was funded by the GINOP 2.3.2-15-2016-00046 project, the Ministry of Human Resources (EFOP-3.6.3-VEKOP-16-2017-00005) and the Hungarian Academy of Sciences (Eötvös Lóránd Research Network MTA-KE-SZIE Mycotoxins in the Food Chain Research Group; 13003).

Institutional Review Board Statement: The study was conducted in accordance with the Declaration of Helsinki, and the protocol was approved by the Scientific and Research Ethical Committee of the Medical Research Council (Hungary) (ID 32958-3/2017/EKU), and included informed, written consent of all patients.

Informed Consent Statement: Informed consent was obtained from all subjects involved in the study.

Data Availability Statement: The data that support the findings of this study are available from the corresponding author upon reasonable request. 
Acknowledgments: Moritz Kaposi Teaching General Hospital participated in the sample collection and determined the creatinine concentration, for which we are grateful. We would like to thank Antonio Logrieco for his professional support.

Conflicts of Interest: The authors declare no conflict of interest.

\section{References}

1. Turner, P.C.; Flannery, B.; Isitt, C.; Ali, M.; Pestka, J. The role of biomarkers in evaluating human health concerns from fungal contaminants in food. Nutr. Res. Rev. 2012, 25, 162-179. [CrossRef] [PubMed]

2. Turner, P.C.; Snyder, J.A. Development and Limitations of Exposure Biomarkers to Dietary Contaminants Mycotoxins. Toxins 2021, 13, 314. [CrossRef]

3. Yazar, S.; Omurtag, G.Z. Fumonisins, trichothecenes and zearalenone in cereals. Int. J. Mol. Sci. 2008, 9, 2062-2090. [CrossRef] [PubMed]

4. Solfrizzo, M.; Gambacorta, L.; Visconti, A. Assessment of multi-mycotoxin exposure in Southern Italy by urinary multi-biomaker determination. Toxins 2014, 6, 523-538. [CrossRef] [PubMed]

5. Gerding, J.; Cramer, B.; Humpf, H.U. Determination of mycotoxin exposure in Germany using an LC-MS/MS multibiomarker approach. Mol. Nutr. Food Res. 2014, 58, 2358-2368. [CrossRef] [PubMed]

6. Heyndrickx, E.; Sioen, I.; Huybrechts, B.; Callebaut, A.; De Henauw, S.; De Saeger, S. Human biomonitoring of multiple mycotoxins in the Belgian population: Results of the BIOMYCO study. Environ. Int. 2015, 84, 82-89. [CrossRef] [PubMed]

7. Mitropoulou, A.; Gambacorta, L.; Lemming, E.W.; Solfrizzo, M.; Olsen, M. Extended evaluation of urinary multi-analyses of mycotoxins in Swedish adults and children. World Mycotoxin J. 2018, 11, 647-659. [CrossRef]

8. Franco, L.T.; Petta, T.; Rottinghaus, G.; Bordin, K.; Gomes, G.A.; Alvito, P.; Assunção, R.; Oliveira, C.A.F. Assessment of mycotoxin exposure and risk characterization using occurrence data in foods and urinary biomarkers in Brazil. Food Chem. Toxicol. 2019, 128, 21-34. [CrossRef] [PubMed]

9. Lemming, E.W.; Montes, A.M.; Schmidt, J.; Cramer, B.; Humpf, H.U.; Moraeus, L.; Olsen, M. Mycotoxins in blood and urine of Swedish adolescents-Possible associations to food intake and other background characteristics. Mycotoxin Res. 2019, 36, 193-206. [CrossRef]

10. Gambacorta, L.; Solfrizzo, M.; Visconti, A.; Powers, S.; Cossalter, A.M.; Pinton, P.; Oswald, I.P. Validation study on urinary biomarkers of exposure for aflatoxin B1, ochratoxin A, fumonisin B1, deoxynivalenol and zearalenone in piglet. World Mycotoxin J. 2013, 6, 299-308. [CrossRef]

11. Shephard, G.S.; Burger, H.M.; Gambacorta, L.; Gong, Y.Y.; Krska, R.; Rheeder, J.P.; Solfrizzo, M.; Srey, C.; Sulyok, M.; Visconti, A.; et al. Multiple mycotoxin exposure determined by urinary biomarkers in rural subsistence farmers in the former Transkei, South Africa. Food Chem. Toxicol. 2013, 62, 217-225. [CrossRef] [PubMed]

12. Warth, B.; Sulyok, M.; Berthiller, F.; Schuhmacher, R.; Krska, R. New insights into the human metabolism of the Fusarium mycotoxins deoxynivalenol and zearalenone. Toxicol. Lett. 2013, 220, 88-94. [CrossRef] [PubMed]

13. Turner, P.C.; White, K.L.; Burley, V.J.; Hopton, R.P.; Rajendram, A.; Fisher, J.; Cade, J.E.; Wild, C.P. A comparison of deoxynivalenol intake and urinary deoxynivalenol in UK adults. Biomarkers 2010, 15, 553-562. [CrossRef] [PubMed]

14. Vidal, A.; Claeys, L.; Mengelers, M.; Vanhoorne, V.; Vervaet Chuybrechts, B.; De Saeger, S.; De Boevre, M. Humans significantly metabolize and excrete the mycotoxin deoxynivalenol and its modified form deoxynivalenol-3-glucoside within 24 hours. Sci. Rep. 2018, 8, 5255. [CrossRef] [PubMed]

15. Riley, R.T.; Torres, O.; Showker, J.L.; Zitomer, N.C.; Matute, J.; Voss, K.A.; Gelineau-van Waes, J.; Maddox, J.R.; Gregory, S.G.; Ashley-Koch, A.E. Kinetics of Urinary Fumonisin B1 Excretion in Humans Consuming Maize-Based Diets. Mol. Nutr. Food Res. 2012, 56, 1445-1455. [CrossRef]

16. Fan, K.; Xu, J.; Jiang, K.; Liu, X.; Meng, J.; Di Mavungu, J.D.; Guo, W.; Zhang, Z.; Jing, J.; Li, H.; et al. Determination of multiple mycotoxins in paired plasma and urine samples to assess human exposure in Nanjing, China. Environ. Pollut. 2019, 248, 865-873. [CrossRef] [PubMed]

17. Zhu, J.; Zhang, L.; Hu, X.; Urine, H.; Xiao, Y.; Chen, J.; Xu, Y.; Fremy, J.; Chu, F.S. Correlation of dietary aflatoxin B1 levels with excretion of aflatoxin M1 in human urine. Cancer Res. 1987, 47, 1848-1852. [PubMed]

18. Degen, G.H. Are we ready to estimate daily ochratoxin A intake based on urinary concentrations? Environ. Int. 2016, 97, 254-255. [CrossRef] [PubMed]

19. Gambacorta, L.; Magistà, D.; Perrone, G.; Murgolo, S.; Logrieco, A.F.; Solfrizzo, M. Co-occurrence of toxigenic moulds, aflatoxins, ochratoxin A, Fusarium and Alternaria mycotoxins in fresh sweet peppers (Capsicum annuum) and their processed products. World Mycotoxin J. 2018, 11, 159-173. [CrossRef]

20. Solfrizzo, M.; Gambacorta, L.; Lattanzio, V.M.T.; Powers, S.; Visconti, A. Simultaneous LC-MS/MS determination of aflatoxin M1, ochratoxin A, deoxynivalenol, de-epoxydeoxynivalenol, $\alpha$ and $\beta$-zearalenols and fumonisin B1 in urine as a multi-biomarker method to assess exposure to mycotoxins. Anal. Bioanal. Chem. 2011, 401, 2831. [CrossRef]

21. Studer-Rohr, I.; Schlatter, J.; Dietrich, D.R. Kinetic parameters and intraindividual fluctuations of OTA plasma levels in humans. Arch. Toxicol. 2000, 74, 499-510. [CrossRef] 
22. EFSA. Appropriateness to set a group health-based guidance value for zearalenone and its modified forms. EFSA J. $2016,14,4425$. [CrossRef]

23. EFSA. Appropriateness to set a group health-based guidance valuefor fumonisins and their modified forms. EFSA J. 2018, 16, 5172. [CrossRef]

24. EFSA. Opinion of the scientific panel on contaminants in the food chain on a request from the commission related to ochratoxin A in food. EFSA J. 2006, 365, 1-56. [CrossRef]

25. Van der Westhuizen, L.; Shepard, G.; Burger, H.M.; Rheeder, J.P.; Gerderblom, W.C.A.; Wild, C.P.; Gong, Y.Y. Fumonisin B1 as a urinary biomarker of exposure in a maize intervention study among South African subsistence farmers. Cancer Epidemiol. Biomark. Prev. 2011, 20, 483-489. [CrossRef] [PubMed]

26. D'Arco, G.; Fernández-Franzón, M.; Font, G.; Damiani, P.; Mañes, J. Survey of fumonisins B1, B2 and B3 in conventional and organic retail corn products in Spain and Italy and estimated dietary exposure. Food Addit. Contam. Part B. 2019, 2, 146-153. [CrossRef]

27. Schlatter, C.; Studer-Rohr, J.; Rasonyi, T. Carcinogenicity and kinetic aspects of ochratoxin A. Food Addit. Contam. 1996, 13, 43-44. 